\title{
CRYOGENIC INFRASTRUCTURE FOR FERMILB'S ILC VERTICAL CAVITY TEST FACILITY
}

\author{
R. Carcagno, C. Ginsburg, Y. Huang, B. Norris, J. Ozelis, T. Peterson, \\ V. Poloubotko, R. Rabehl, C. Sylvester, M. Wong
}

Fermi National Accelerator Laboratory

Batavia, IL, 60510, USA

\begin{abstract}
Fermilab is building a Vertical Cavity Test Facility (VCTF) to provide for R\&D and pre-production testing of bare 9-cell, 1.3-GHz superconducting RF (SRF) cavities for the International Linear Collider (ILC) program. This facility is located in the existing Industrial Building 1 (IB1) where the Magnet Test Facility (MTF) also resides. Helium and nitrogen cryogenics are shared between the VCTF and MTF including the existing 1500-W at 4.5-K helium refrigerator with vacuum pumping for super-fluid operation (125-W capacity at 2-K). The VCTF is being constructed in multiple phases. The first phase is scheduled for completion in mid 2007, and includes modifications to the IB1 cryogenic infrastructure to allow helium cooling to be directed to either the VCTF or MTF as scheduling demands require. At this stage, the VCTF consists of one Vertical Test Stand (VTS) cryostat for the testing of one cavity in a 2-K helium bath. Planning is underway to provide a total of three Vertical Test Stands at VCTF, each capable of accommodating two cavities. Cryogenic infrastructure improvements necessary to support these additional VCTF test stands include a dedicated ambient temperature vacuum pump, a new helium purification skid, and the addition of helium gas storage. This paper describes the system design and initial cryogenic operation results for the first VCTF phase, and outlines future cryogenic infrastructure upgrade plans for expanding to three Vertical Test Stands.
\end{abstract}

KEYWORDS: Test facilities, SRF cavities, Controls

\section{INTRODUCTION}

The Vertical Cavity Test Facility (VCTF) at Fermilab is designed to test superconducting RF (SRF) cavities at a nominal frequency of $1.3-\mathrm{GHz}$ in a $2-\mathrm{K}$ liquid 
helium bath [1], and initially will support up to 48 cavity tests per year. Cavity performance is measured in order to evaluate cavity processing methods and procedures, and also as an acceptance test prior to installation of additional cavity components such as tuners and helium vessels, prior to testing in a horizontal cryostat.

The VCTF has been designed for SRF cavity tests measuring low-field cavity quality factor (Q) vs. temperature down to approximately 1.6-K, and Q vs. accelerating gradient $\left(E_{\text {acc }}\right)$ at 2.0-K. The facility currently consists of one Vertical Test Stand (VTS), which houses a new cryostat fabricated by PHPK Technologies of Columbus, Ohio. The cavity cooling is provided by the existing Industrial Building 1 (IB1) cryoplant, capable of delivering LHe to the test stand at a peak rate of 700 liters/hr and maintaining a bath temperature of 2-K with up to $125-\mathrm{W}$ of continuous power dissipation into the He bath [2].

The RF system is based on that of Jefferson Lab's vertical test facility, modified for 1.3-GHz operation and taking advantage of technology advances. Cavities in the cryostat will be magnetically shielded, to avoid increasing surface resistance due to trapped magnetic flux in localized normal-conducting surface impurities. In addition, cavities will be radiation shielded, to maintain the "controlled area" status of IB1, i.e., integrated dose rates $<5 \mathrm{mrem} / \mathrm{hr}$ in the immediate vicinity of the shielding, and $<0.25 \mathrm{mrem} / \mathrm{hr}$ in normal working areas.

The first VTS is in the commissioning stages and will be fully operational by October, 2007. Two additional test stands and cryogenic upgrades are being planned for installation during the 2008-2009 timeframe. These upgrades will provide VCTF with a cavity test throughput of approximately 264 tests per year.

\section{DESCRIPTION OF IB1 CRYOGENICS AND TEST STAND SUPPORT}

The IB1 cryogenic system consists of a 1500-W helium refrigerator, a helium distribution box system and Kinney vacuum pumping skids. The helium plant is comprised of a two stage helium compressor skid, a cold-box, a 10,000-liter liquid helium storage Dewar, three $114-\mathrm{m}^{3}$ gas helium buffer tanks and a partial helium gas purification system to scrub $10-20 \%$ of gas helium returned from all cold test stands. A block diagram of the existing cryogenic infrastructure is shown in FIGURE 1 . The plant runs nominally as a liquefier continuously supporting the cold test stands in IB1 in the temperature range from 1.8-K to $4.5-\mathrm{K}$.

Currently the cryogenic infrastructure in IB1 is primarily used to support Tevatron and high field magnet tests in the temperature range from $1.8-\mathrm{K}$ to $4.5-\mathrm{K}$. For Tevatron magnets, the desired temperature range is $3.6-\mathrm{K}$ to $4.5-\mathrm{K}$ with forced flow single phase helium in the magnet cold mass and saturated two phase helium flowing in the opposite direction to provide sub-cooling to the single phase. LARP/HFM magnets, (either dipole or quads), are tested in the Vertical Magnet Test Facility (VMTF). This test stand consists of a vertical test dewar of $655.6-\mathrm{mm}$ in diameter and 5205-mm deep. The magnet test temperature ranges are $1.8-\mathrm{K}$ to $4.5-\mathrm{K}[3]$.

\section{DESCRIPTION OF VTS PHASE 1}

\section{Cryostat}

The VTS cryostat consists of an ASME code-stamped helium vessel (rated to 552-kPa) within a vacuum vessel, both manufactured from type 304 stainless steel. The vacuum 


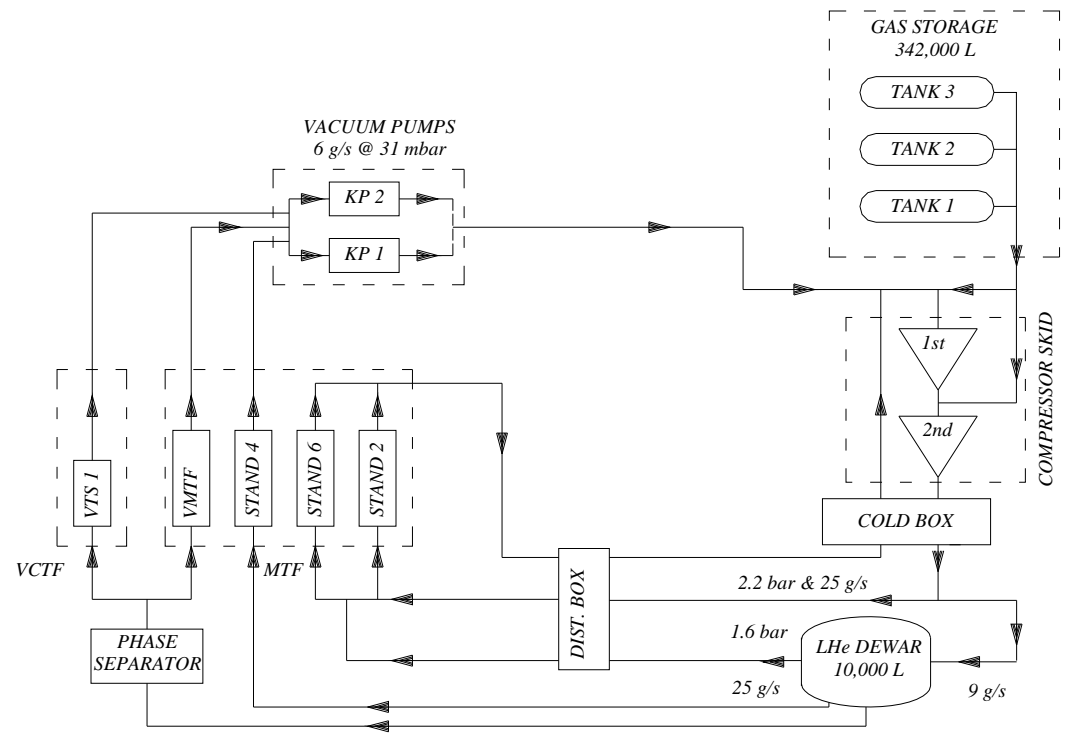

FIGURE 1. IB1 Cryogenic infrastructure and test stand support.

vessel has a 1-m inner diameter, 9.5-mm wall thickness and is 4.9-m long. Two-phase helium is supplied to the cryostat through a phase separator, and enters the helium vessel either through a top-fill or bottom-fill port. A heater located at the bottom of the helium vessel provides up to 250-W to expedite LHe boil-off and warm-up of the Dewar after cavity testing is complete. Another heater located in the phase separator provides $5-10$ watts of power to generate a gas supply for the 5-K baffle

The cryostat is equipped with redundant ASME-compliant pressure relief systems for the helium circuit, which is comprised of a primary device that includes a 3.8-cm diameter burst disk combined with a re-closable safety relief valve, at a set pressure of $483-\mathrm{kPa}$. A similar device configured in parallel, having a set pressure of 552-kPa, acts as a secondary relief. The relief system is sized assuming complete helium vaporization due to a leak in the insulating vacuum, resulting in a heat flux of $0.6-\mathrm{W} / \mathrm{cm}^{2}$ on the helium vessel wall [4]. For operational pressure relief, a Fermilab-built Kautzky relief valve with a set pressure of $205-\mathrm{kPa}$ is installed in parallel with these relief systems. All three devices are designed to avoid sub-atmospheric leakage into the helium circuits.

To reduce the cryostat's overall static heat load at $2-\mathrm{K}$, an $80-\mathrm{K}$ thermal shield is mounted between the helium and vacuum vessel walls, and a $5-\mathrm{K}$ thermal intercept is welded to the exterior of the helium vessel.

\section{Magnetic Shielding}

To avoid trapping magnetic flux and thereby degrading the cavity quality factor, the cavity must be shielded from magnetic fields during cool down. This is accomplished using a two-layer cylindrical magnetic shielding design, expected to yield a remnant field of < $10 \mathrm{mG}$ in the cavity region. The design incorporates a room temperature outer shield outside the vacuum vessel, and an inner shield inside the helium vessel. The outer shield is made of $1 \mathrm{~mm}$ thick Amumetal ${ }^{\circledR}$, with a length of $4200-\mathrm{mm}$ and an inner diameter of 1070 $\mathrm{mm}$. The inner shield has one perforated end cap at the bottom (for LHe flow), and is made of 1-mm thick Cryoperm $10^{\circledR}$, with a length of 3378-mm and an inner diameter of 635-mm. It is attached to the outer surface of an aluminum cylinder for support and protection, and will be permanently installed inside the cryostat. 
The magnetic field inside the cryostat has been measured as a function of depth, and at various azimuthal positions. Measurements were performed in the empty pit and in the helium vessel of the cryostat with and without the outer shielding in place. The measurement in the empty pit was consistent with the Earth's magnetic field. The measurement with the cryostat and outer shield in place shows that the field in the area of interest is less than $50 \mathrm{mG}$. The measurement of the magnetic field with both shields in place will be done soon.

\section{Test Stand Insert}

The test stand insert supports the cavity in the LHe bath. It consists of a 31.75-mm thick type 304 stainless steel top plate with several penetrations for RF and instrumentation cable feedthroughs. A set of thermal radiation baffles and a 5-K helium intercept plate are suspended from the top plate using threaded rods, which also support the internal radiation shielding from which the cavity support cage is suspended. A set of G-10 plates located above and below the cavity support cage serve as a standoff for the cavity structure, guiding the insert into the Dewar and protecting the cavity from contact with the Dewar walls. The complete insert with cavity is shown schematically in FIGURE 2.

\section{Internal and External Radiation Shielding}

In order to prevent radiation exposure to personnel a system of radiation shielding has been designed based on modeling using MARS15 [5]. The exposure reduction is achieved using both internal radiation shielding (mounted on each test stand insert), and an external shield placed over the Dewar top plate during cavity testing.

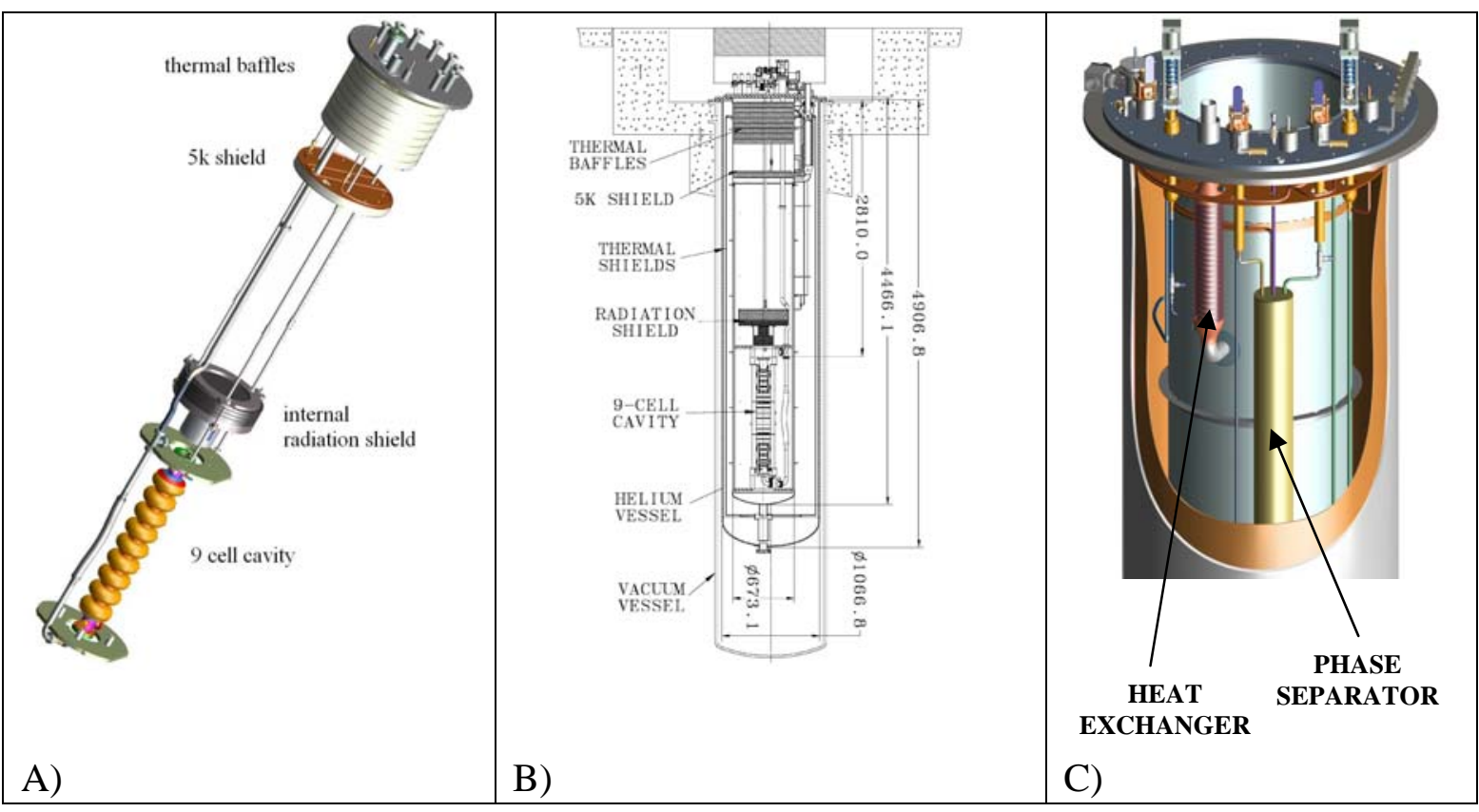

FIGURE 2. A) Complete Insert with Cavity; B) Layout of VTS (dimensions in mm) including the dewar, vacuum vessel, insert with cavity; C) Cutaway of cryostat showing heat exchanger and phase separator. 
The internal shielding consists of a pair of 100-mm thick lead disks (one 200-mm diameter, another 150-mm mounted just above the cavity), a 50.8-mm thick, 533-mm diameter steel disk mounted above the lead disk, and a 101.6-mm thick, 533-mm diameter disk of borated polyethylene above the steel disk. The mass of the Pb shielding equals approximately 60-kg, while the steel shielding has a mass of about 90-kg; these are both significantly greater than the 30-kg weight of the 9-cell cavity to be tested, and as such have a large impact on cooldown and warm-up rate. As this internal shielding is by definition in the LHe bath, consideration must be given to the impact of this substantial thermal mass on cryogenic operations, especially cool down and warm-up times. Calculations show that the mass of the internal shielding entails an additional 11.4-MJ of heat that needs to be removed from the system during cool down, compared to the 1.5-MJ due solely to the cavity to be tested.

The external shielding comprises a movable lid and chain drive system. The lid moves on rails mounted to the concrete floor of the building. The rails can be extended to allow this shield to service future additional in-ground Dewars. The lid provides radiation absorption by utilizing a 152.4-mm thick steel base and 457.2-mm high concrete blocks. The base has cutouts to accept Hillman ${ }^{\circledR}$ heavy-duty rollers, which provide a low friction system for movement of this 28122-kg assembly. The chain pull system consists of a chain drive motor and gearbox, and two chain channels with idler sprockets that guide the floormounted chains over the radiation lid as it moves. A cable gantry and wall-mounted track provides the means for supplying power to the shielding block mounted motor controller as it moves. A 3-D model of this design is given in FIGURE 3.

\section{Cryogenic System and Controls}

Cryogenic process controls for the VTS are based on controls used at the superconducting magnet test facility cryoplant which is shared by the VTS [6]. Two layers of control are implemented - a PLC at the field level and a commercial SCADA/HMI for operator interfacing. A Siemens 545 Simatic PLC unit connects to approximately 70

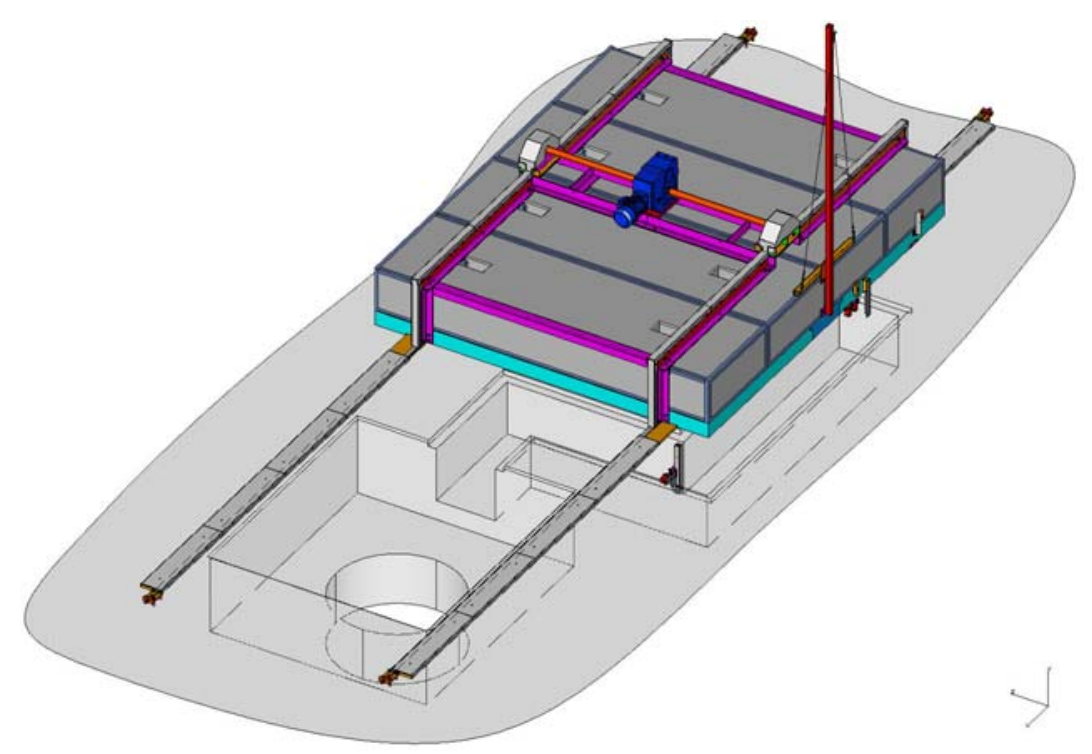

Figure 3. Mechanical design of the radiation shielding lid. The lid is moved over the VTS pit for RF testing. 
sensors for monitoring and controlling all stages of the cryogenic process. Sensotec and MKS transducers are used to monitor pressure, LHe level monitoring is performed by American Magnetics Model 135-2K instruments, while Lakeshore Cernox CX-1030-SD devices allow bath temperatures to be measured down to the 1.6-K design point. Control loops are established for level and pressure control inside of the PLC logic.

GE Fanuc's Intellution iFix 3.5 SCADA systems, already in use for cryogenic plant operations, has been extended for all cryogenic process data logging and control of the VTS. Cryogenic plant operators can control the plant and any test stand, including VTS, from one central control room. The overall control strategy for VTS is to maximize automation and minimize operator intervention. Each cavity tested in the VTS will require a set of cryogenic operations - including pump and backfill, contamination monitoring, 4.5-K fill, 2-K pump down, and warm up - which will be automated as batch processes. Once their systematic behavior is verified and proven to be repeatable, this strategy will be fully implemented.

\section{Initial Cryogenic Performance}

The VTS began successful cryogenic commissioning in May 2007 and has been successfully integrated into the IB1 refrigeration complex as a valid stand for testing ILC SRF 9-cell cavities. During the initial cool down, after about an hour of helium flow liquid helium began to collect in the cryostat at a rate of about 500-L/hr (16.7-g/sec), consistent with expectations. Static heat leak into the helium bath was measured, agreeing well with the calculated value of $4.6-\mathrm{W}$.

The helium bath was subsequently pumped down to 2-K (31-mbar) over a period of just over 3 hours, at which point a helium level of 2000-mm ( 700-L) remained in the cryostat. This pump down speed compares very favorably with other similar facilities. The lowest bath temperature reached was $1.55-\mathrm{K}$ which is limited by the vacuum pump inlet pressure. The pump down curve is shown in FIGURE 4.

\section{DESCRIPTION OF FUTURE VTS PHASES}

Increasing the number of cryostats to three, each equipped with two cavities, and implementing IB1 infrastructure upgrades increases the VCTF throughput to a maximum

of 264 cavity tests per year. The infrastructure upgrades include a new purifier, a dedicated ambient temperature 2-K pumping system, and additional gas storage. Each upgrade is highlighted here in order of priority. FIGURE 5 shows the proposed changes.

\section{Adding a Full Flow Purifier}

Scheduling risk and refrigerator downtime will be reduced by installing a compressor/purifier skid at the outlet of the vacuum pumps to eliminate contamination from sub-atmospheric operations (the main source of contamination due to air leaks). A compressor is needed to increase the effectiveness of purification from the absorber beds by increasing the GHe pressure. As stated previously, the existing IB1 cryogenic system has a partial flow purifier that can only purify 10 to $20 \%$ of the cold box inlet flow. 


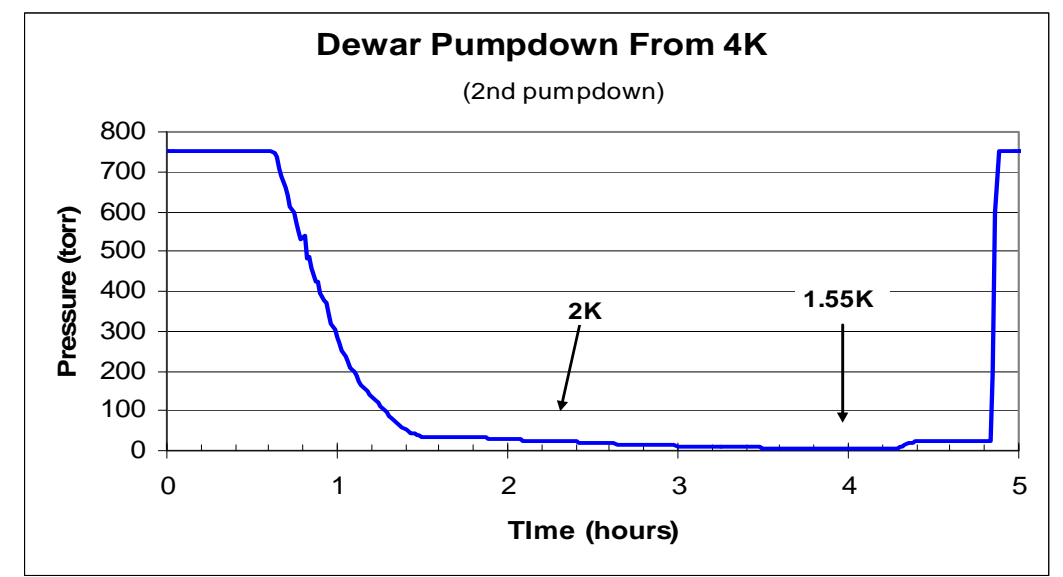

FIGURE 4. Initial Pump down of VTS.

\section{Adding a Dedicated Vacuum Pump}

The existing 4-g/sec and 2-g/sec (at 31-mbar) vacuum pumps used for VTS are currently shared with the magnet test facility, and the resulting interference between magnet and cavity tests is expected to cause scheduling inefficiency. Installing a dedicated vacuum pump for the VTS will eliminate this strong coupling, permitting superfluid magnet testing to proceed in parallel with cavity testing.

\section{Adding Gas Storage}

The helium gas storage capacity of the IB1 cryogenic system is limited to about half of the liquid storage capacity, which limits use of the stored liquid. An upper limit on the buffer tank pressure is necessary to maintain control of the compressor skid pressures via the gas management system. Once this pressure limit is reached, testing must be halted regardless of

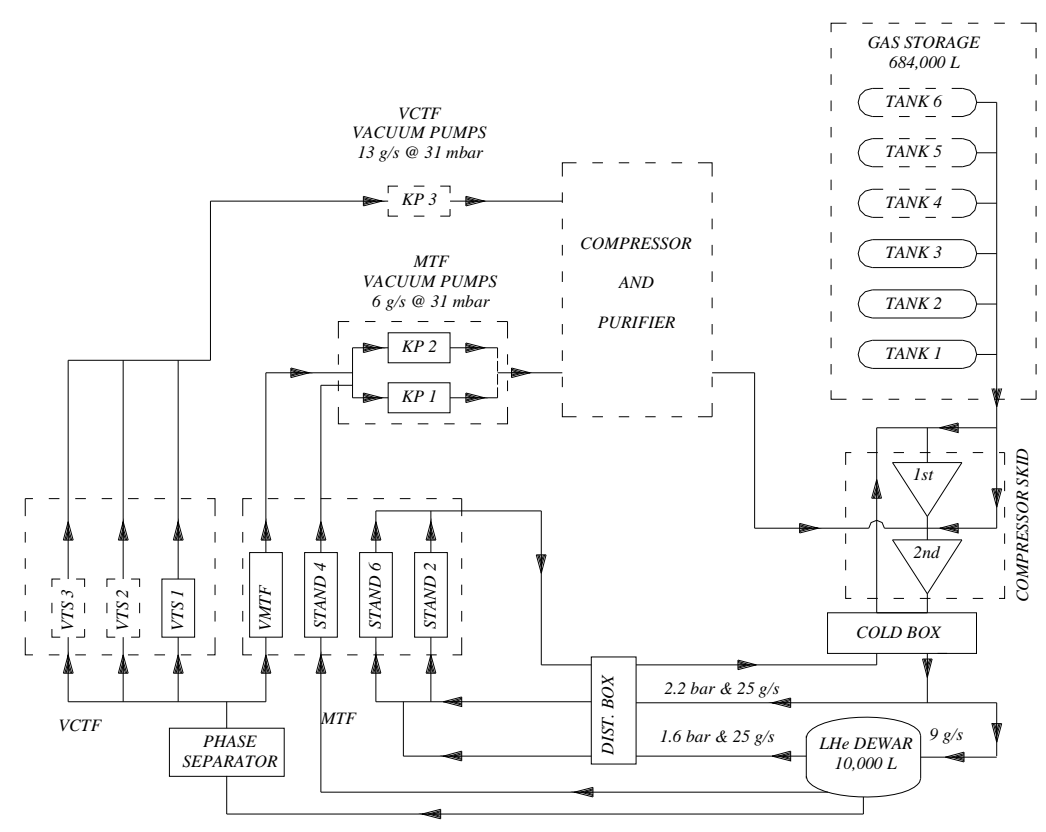

FIGURE 5. Future IB1 cryogenic infrastructure and test stand support. 
the liquid inventory in order to re-liquefy helium and lower the buffer tank pressure. The addition of three more $114-\mathrm{m}^{3}$ buffer tanks will increase gas storage volume and would allow the cryogenic system to support longer periods of testing.

\section{SUMMARY}

A new facility for testing ILC SRF cavities at a nominal frequency of $1.3-\mathrm{GHz}$ in a 2.0-K bath has been cryogenically commissioned in May 2007. The first Vertical Test Stand has been successfully integrated into the cryogenic infrastructure of Fermilab's IB1 facility which is supporting a magnet test program as well. Initial cryogenic performance has proven to verify theoretical calculations such as nominal $4-\mathrm{K}$ and $2-\mathrm{K}$ heat leak, liquid fill rates, and pump down capacity to $2-\mathrm{K}$ from $4.2-\mathrm{K}$. The cryogenic controls have been seamlessly integrated with the original plant controls and give operators the tools they need to take a test cavity through complete cryogenic cycles.

In July and August 2007, this facility will be fully commissioned in every phase and will begin to be used daily to evaluate cavity processing methods as well as allow cavity acceptance tests prior to adding tuners and helium vessels.

Work in FY2008 and FY2009 will concentrate on infrastructure upgrades focused on increasing the number of cavity tests per year. Two more vertical test stands will be added, cryogenic improvements including a new dedicated vacuum pump, a full flow purifier, and more gas storage will make IB1 a premiere U. S. facility for critical ILC cavity tests.

\section{ACKNOWLEDGEMENTS}

This work is supported by the U. S. Department of Energy under contract No DEAC02-07CH11359.

\section{REFERECENCES}

1. Ozelis, J. P., et al., "Design and Commissioning of Fermilab’s Vertical Test Stand for ILC SRF Cavities”, presented at the 2007 Particle Accelerator Conference.

2. Huang, Y. and Ozelis, J. P., "Performance Analysis and Requirements of the ILC Vertical Cavity Test Stand Cryogenic System at IB1”, Fermilab note TD-06-012.

3. Peterson, T.J., et al., “A 1400 Liter 1.8K Test Facility,” Advances in Cryogenic Engineering, vol. 43a, pp. 541-548, Plenum Press, New York, (1997).

4. Lehmann, W. et al, "Safety Aspects for LHe Cryostats and LHe Transport Containers,” Proceeding of the $7^{\text {th }}$ International Cryogenic Engineering Conference, London, 1978.

5. Rakhno, I., "Raditiation Shielding Issues for Superconducting RF Cavity Test Facility at Fermilab”, Fermilab note TM-2367-AD.

6. Carcagno R. and Rabehl R., "Controls Upgrade of the Fermilab Magnet Test Facility 1500 W Helium Refrigerator,” Advances in Cryogenic Engineering, vol. 45, pp. 1795-1802, Plenum Press, New York, (2000). 